\title{
Non-Oscillating solutions of a generalized system of ODEs with derivative terms
}

https://doi.org/10.1515/msds-2020-0136

Received April 28, 2021; accepted October 19, 2021

Abstract: We consider a system of ODEs of mixed order with derivative terms appearing in the non-linear function and show the existence of a solution which does not oscillate for such system. We applied the fixed point technique to show that under certain conditions there exists at least one solution to the system which is not only non-oscillating, but also asymptotically constant.

Keywords: Ordinary Differential Equations, Fixed-point Theorem, Non-Oscillation

MSC: 34C11, 45P05, 46N20

\section{Introduction}

Systems of differential equations arise while modelling many situations. We will now look at few systems of coupled ODEs which arise in nature. In [1], the authors discuss some qualitative properties of elliptic systems of the type

$$
\begin{array}{ccc}
\Delta u+a(|x|) f(u, v) & = & 0, \\
\Delta v+b(|x|) g(u, v) & = & 0, \\
R_{1}<|x|<R_{2}, & x \in \mathbb{R}^{n} .
\end{array}
$$

They considered the existence of positive radial solutions for this system. In view of the spherical symmetry of $g(|x|)$, they rewrite the system (1.2) and find positive solutions for

$$
\begin{aligned}
& u^{\prime \prime}(r)+\frac{N-1}{r} u^{\prime}(r)+a(r) f(u(r), v(r))=0, \\
& v^{\prime \prime}(r)+\frac{N-1}{r} v^{\prime}(r)+b(r) g(u(r), v(r))=0 .
\end{aligned}
$$

The following system of ODEs appears in Non-linear Optics [11]:

$$
\begin{aligned}
& -w^{\prime \prime}+a w-w v=0, \\
& -v^{\prime \prime}+b v-\frac{w^{2}}{2}=0,
\end{aligned}
$$

where $a, b$ are positive constants and $w, v$ are functions defined for all $x \in \mathbb{R}$. This system arises from a system of $\chi^{2}-\mathrm{SHG}$ equations which govern phenomena in non-linear optics. The $\chi^{2}-\mathrm{SHG}$ equations arise while studying the Parametric interactions of intense light signals in materials with second-order non-linearities. It is very important to know the asymptotic behaviour of solutions of such systems. In fact, Lopes in [11] talks about solutions of (1.4) with finite energy (equivalently tending to zero as the independent

\footnotetext{
*Corresponding Author: B V K Bharadwaj: Department of Mathematics and Computer Science, Sri Sathya Sai Institute of Higher Learning, Prasanthi Nilayam, INDIA, E-mail: bvkbharadwaj@sssihl.edu.in

Pallav Kumar Baruah: Department of Mathematics and Computer Science, Sri Sathya Sai Institute of Higher Learning, Prasanthi Nilayam, INDIA, E-mail: pkbaruah@sssihl.edu.in
} 
variable say $x \rightarrow \infty$ ).

While considering the steady flow of an electrically conducting fluid between two horizontal parallel plates where the fluid and the plates rotate in unison about an axis normal to the plates with an angular velocity, the following system of ODEs arise.

$$
\begin{aligned}
f^{\prime \prime \prime}-M^{2} f^{\prime}-2 K^{2} g & =R\left[\left(f^{\prime}\right)^{2}-f f^{\prime}\right]+A, \\
g^{\prime \prime}-M^{2} g+2 K^{2} f^{\prime} & =R\left[f^{\prime} g-f g\right],
\end{aligned}
$$

where $A$ is a constant, $R$ is the viscosity parameter, $M^{2}$ is the magnetic parameter and $K^{2}$ is the rotational parameter. The functions $f$ and $g$ are defined on the real line. These equations are found in [12].

These examples lead us to consider the more generalized forms of (1.3). So, we consider the following generalized version of coupled system of non-linear ordinary differential equations.

$$
\begin{aligned}
& x_{1}^{\left(n_{1}\right)}(t)+h_{1}\left(t, x_{1}, x_{1}^{\prime}, . ., x_{1}^{\left(N_{1}\right)}, x_{2}, x_{2}^{\prime}, . ., x_{2}^{\left(N_{2}\right)}\right)=0, \\
& x_{2}^{\left(n_{2}\right)}(t)+h_{2}\left(t, x_{1}, x_{1}^{\prime}, . ., x_{1}^{\left(N_{1}\right)}, x_{2}, x_{2}^{\prime}, . ., x_{2}^{\left(N_{2}\right)}\right)=0,
\end{aligned}
$$

where $h_{1}, h_{2}$ are real valued continuous functions on $\left[t_{0}, \infty\right) \times \mathbb{R}^{N_{1}+N_{2}}, t \geq t_{0}>0, n_{i}>1$ and $N_{i}$ are integers with $0 \leq N_{i} \leq n_{i}-1$ for $i=1$, 2 .

Lot of work is done in providing conditions to find non oscillating solutions of systems and equations. We refer to articles like $[2-4,6,7,9,14$ ? ]. In most of these article authors proved conditions for non oscillation or positive solutions for either lower order systems which are fully coupled or higher order systems which are weakly coupled. For example, Graef et al [13] considered the following second order system which is fully coupled.

$$
\begin{aligned}
& -u_{1}^{\prime \prime}=\lambda_{1} a_{1}(t) f_{1}(u, v) \quad t \in(0,1), \\
& -v_{1}^{\prime \prime}=\lambda_{2} a_{2}(t) f_{2}(u, v) \quad t \in(0,1) .
\end{aligned}
$$

Whereas Henderson et al [14] considered the following higher order system.

$$
\begin{aligned}
& u^{(n)}(t)+\lambda a(t) f(v)=0 \quad t \in(0,1), \\
& v^{(n)}(t)+\lambda b(t) g(u)=0 \quad t \in(0,1) .
\end{aligned}
$$

To the best of our knowledge more general systems of the type (1.5) are not considered in existing literature, which makes this problem worth attempting. In this article we presented different conditions under which the existence of a non-oscillating solution for system (1.5) is guaranteed. At the end we apply the theory developed on a theoretical example.

\section{Mathematical Preliminaries}

We present here some mathematical preliminaries required to prove the main results presented in the next section.

Definition 2.1. A pair of real valued continuous functions $x_{1}(t), x_{2}(t)$ are called solutions of (1.5) if they satisfy both the equations in (1.5) simultaneously on the interval $\left[t_{0}, \infty\right)$.

Definition 2.2. A continuous real valued function defined on $\left[t_{0}, \infty\right)$ is said to be oscillatory if it has arbitrarily large zeros and otherwise it is said to be non oscillatory. A solution $\left(x_{1}, x_{2}\right)$ of the system (1.5) is called oscillatory if both $x_{1}$ and $x_{2}$ are oscillatory. If a system has at least one non-trivial solution with finitely many zeros, then it will be called non-oscillatory. 
We will now state Shauder's theorem

Let $E$ be a Banach space and $X$ any nonempty convex and closed subset of $E$. If $S$ is a continuous mapping of $X$ into itself and $S X$ is relatively compact, then the mapping $S$ has at least one fixed point (i.e. there exists an $x \in X$ with $x=S x$ ).

Consider $B([0, \infty))$ to be the Banach space of all continuous and bounded real valued functions on the interval $[0, \infty]$, endowed with the sup-norm $\|\cdot\|$ :

$\|h\|=\sup _{t \geq 0}|h(t)|$ for $h \in B([0, \infty))$

We now define the set $(B)^{N}([0, \infty))$ as:

$(B)^{k}([0, \infty))$ is the set of all bounded continuous real valued functions on the interval $[0, \infty)$ which have bounded continuous $k$-order derivatives on $[0, \infty)$ for each $k=1,2, \ldots, N$.

Let us now define $\|\cdot\|_{N}$ as

$$
\|h\|_{N}=\max _{k=0,1, . ., N}\left\|h^{(k)}\right\|
$$

for $h \in(B)^{N}([0, \infty))$ We note that $(B)^{N}([0, \infty))$ is complete under the norm $\|\cdot\|_{N}$.

Let $(B)^{N}([0, \infty)) \times(B)^{N}([0, \infty))$ be the Cartesian product of $(B)^{N}([0, \infty))$ with itself with the product topology. This product space is endowed with the norm $\|\cdot\|_{N \times N}$ defined as

$$
\left\|\left(u_{1}, u_{2}\right)\right\|_{N \times N}=\max \left\{\left\|u_{1}\right\|_{N},\left\|u_{2}\right\|_{N}\right\} .
$$

We will now need a criterion to show the compactness of subsets of the space $(B)^{N}([0, \infty])$. In that direction, we will revisit few definitions of concepts related to sets of functions taking real values.

Definition 2.3. A set of real-valued functions defined on the interval $[0, \infty), U$ is called uniformly bounded if there exists a positive constant $M$ such that, for all functions $u \in U$

$$
|u(x)| \leq M
$$

for every $x \geq 0$.

Definition 2.4. $U$ is said to be equicontinuous if, for each $\epsilon>0$, there exists a $\delta \equiv \delta(\epsilon)>0$ such that, for all functions $u \in U$

$$
\left|u\left(t_{1}\right)-u\left(t_{2}\right)\right|<\epsilon
$$

for every $t_{1}, t_{2} \geq 0$ with $\left|t_{1}-t_{2}\right|<\delta$.

Definition 2.5. $U$ is called equiconvergent at $\infty$ if all functions in $U$ are convergent in $R$ at the point $\infty$ and, for each $\epsilon>0$, there exists a $T>0$ such that, for all $u \in U$

$$
\left|u(x)-\lim _{s \rightarrow \infty} u(s)\right|<\epsilon
$$

for every $x \geq T$.

We will use the following lemma from [5] which is a generalization of the above mentioned compactness criterion.

Lemma 2.6. Let $H \times H$ be a subset of the Banach Space $(B)^{N}([0, \infty)) \times(B)^{N}([0, \infty))$ endowed with the norm $\|\cdot\|_{N \times N}$. Define $H^{(0)} \times H^{(0)}=H \times H$ and provided that $N>0, H^{(k)} \times H^{(k)}=\left\{\left(h_{1}^{(k)}, h_{2}^{(k)}\right):\left(h_{1}, h_{2}\right) \in H \times H\right\}$ for $k=1,2, \ldots, N$. If $H^{(k)} \times H^{(k)}(k=0,1,2, . ., N)$ are uniformly bounded, equicontinuous and equiconvergent at $\infty$, then $H \times H$ is relatively compact.

\section{Main Results}

The following is our main theorem. 
Theorem 3.1. Let

$$
\left|h_{i}\left(t, z_{1}, z_{2}, . . z_{N_{1}+N_{2}}\right)\right| \leq \sum_{k=0}^{N_{1}+N_{2}} p_{i k}(t) g_{i k}\left(\left|z_{k}\right|\right)+r_{i}(t)
$$

for all $\left(t, z_{1}, z_{2}, . . z_{N_{1}+N_{2}}\right) \in\left[t_{0}, \infty\right) \times \mathbb{R}^{N_{1}+N_{2}}$ and $i=1,2$, where $p_{i k}$ and $r_{i}(i=1,2),\left(k=1,2, \ldots, N_{1}+N_{2}\right)$ are non-negative continuous real-valued functions on $\left[t_{0}, \infty\right)$ such that for $N=\max \left\{1, n_{1}, n_{2}\right\}$

$$
\begin{gathered}
\int_{t_{0}}^{\infty} t^{N-1} p_{i k}(t) d t<\infty, \\
\int_{t_{0}}^{\infty} t^{N-1} r_{i}(t) d t<\infty
\end{gathered}
$$

for $i=1,2, k=0,1, . ., N_{1}+N_{2}$ and $g_{i}, i=1,2$ are non-negative continuous real-valued functions on $[0, \infty)$ which are not identically zero.

Let there exist positive constants $K_{1}>0, K_{2}>0$ and $T>t_{0}$ such that for

$$
\max _{k=0,1, . ., N_{1}}\left[\sum_{l=1}^{N_{1}+N_{2}} \int_{T}^{\infty}(s-T)^{n_{1}-1-k} p_{1 l}(s) d s \Theta_{1 l}+\int_{T}^{\infty}(s-T)^{n_{i}-1-k} r_{1}(s) d s\right] \leq K_{1}
$$

and

$$
\max _{k=0,1, \ldots, N_{2}}\left[\sum_{l=1}^{N_{1}+N_{2}} \int_{T}^{\infty}(s-T)^{n_{i}-1-k} p_{2 l}(s) d s \Theta_{2 l}+\int_{T}^{\infty}(s-T)^{n_{i}-1-k} r_{2}(s) d s\right] \leq K_{2},
$$

where $\Theta_{1 l}, \Theta_{2 l}$ are defined as

$$
\begin{aligned}
& \Theta_{1 l}=\sup \left\{g_{1 l}(z): 0 \leq z \leq \max \left\{2 K_{1}, 2 K_{2}\right\}\right\}, \\
& \left.\Theta_{2 l}=\sup \left\{g_{2 l}(z)\right): 0 \leq z \leq \max \left\{2 K_{1}, 2 K_{2}\right\}\right\}
\end{aligned}
$$

for $l=0,1,2, \ldots, N_{1}+N_{2}$. Then the system (1.5) has a solution pair $\left\{x_{1}, x_{2}\right\}$ on $[T, \infty)$ such that $x_{1} \rightarrow K_{1}$ and $x_{2} \rightarrow K_{2}$ asymptotically and therefore are non-oscillatory.

Proof. Consider the space $E=(B)^{(N)}([T, \infty))$ with the norm $\|\cdot\|_{N}$ as in $(2.1)$, and define

$$
X=\left\{x \in E:\|x\|_{N} \leq \max \left\{2 K_{1}, 2 K_{2}\right\}\right\} .
$$

Clearly $X$ is a non-empty closed convex subset of $E$.

Now pick $x_{1}$ and $x_{2}$, two arbitrary functions in the set $X$. Then

$$
\begin{aligned}
& g_{1 l}\left(\left|x_{1}(t)\right|\right) \leq \Theta_{1 l}, \\
& g_{2 l}\left(\left|x_{2}(t)\right|\right) \leq \Theta_{2 l}
\end{aligned}
$$

for $l=1,2, . ., N_{1}+N_{2}$. From (3.1) we get

$$
\begin{aligned}
\left|h_{1}\left(t, x_{1}, x_{1}, \ldots, x_{1}^{\left(N_{1}\right)}, x_{2}, x_{2}, \ldots, x_{2}^{\left(N_{2}\right)}\right)\right| & \leq \sum_{l=1}^{N_{1}+N_{2}} \Theta_{1 l} p_{1 l}(t)+r_{1}(t), \\
\left|h_{2}\left(t, x_{1}, x_{1}, . ., x_{1}^{\left(N_{1}\right)}, x_{2}, x_{2}, . ., x_{2}^{\left(N_{2}\right)}\right)\right| \leq & \sum_{l=1}^{N_{1}+N_{2}} \Theta_{2 l} p_{2 l}(t)+r_{2}(t)
\end{aligned}
$$

for every $t \geq T$.

Thus, from (3.2) we conclude that

$$
\left.\int_{T}^{\infty}(s-T)^{N-1} h_{1}\left(t, x_{1}(s), x_{1}^{\prime}(s), . ., x_{1}^{\left(N_{1}\right)}(s), x_{2}(s), x_{2}(s), . ., x_{2}^{\left(N_{2}\right)}(s)\right)\right) d s,
$$




$$
\left.\int_{T}^{\infty}(s-T)^{N-1} h_{2}\left(t, x_{1}(s), x_{1}^{\prime}(s), \ldots, x_{1}^{\left(N_{1}\right)}(s), x_{2}(s), x_{2}^{\prime}(s), \ldots, x_{2}^{\left(N_{2}\right)}(s)\right)\right) d s
$$

exist in $\mathbb{R}$.

Now, by using (3.5), we get that,

$$
\begin{aligned}
& \left.\mid \int_{T}^{\infty}(s-T)^{N-1} h_{1}\left(t, x_{1}(s), x_{1}^{\prime}(s), . ., x_{1}^{\left(N_{1}\right)}(s), x_{2}(s), x_{2}^{\prime}(s), \ldots, x_{2}^{\left(N_{2}\right)}(s)\right)\right) d s \mid \\
& \leq \sum_{l=1}^{N_{1}+N_{2}} \Theta_{1 l} \int_{T}^{\infty}(s-T)^{N} p_{1 l}(s) d s+\int_{T}^{\infty}(s-T)^{N} r_{1}(s) d s, \\
& \left.\mid \int_{T}^{\infty}(s-T)^{N-1} h_{2}\left(t, x_{1}(s), x_{1}^{\prime}(s), \ldots, x_{1}^{\left(N_{1}\right)}(s), x_{2}(s), x_{2}^{\prime}(s), \ldots, x_{2}^{\left(N_{2}\right)}(s)\right)\right) d s \mid \\
& \leq \sum_{l=1}^{N_{1}+N_{2}} \Theta_{2 l} \int_{T}^{\infty}(s-T)^{N-1} p_{2 l}(s) d s+\int_{T}^{\infty}(s-T)^{N} r_{2}(s) d s .
\end{aligned}
$$

From (3.3) and (3.4) for every $t \geq T$ we get

$$
\begin{aligned}
& \left.\mid \int_{t}^{\infty}(s-t)^{N-1} h_{1}\left(t, x_{1}(s), x_{1}^{\prime}(s), . ., x_{1}^{\left(N_{1}\right)}(s), x_{2}(s), x_{2}(s), . ., x_{2}^{\left(N_{2}\right)}(s)\right)\right) d s \mid \\
& \leq K_{1} \text {, } \\
& \left.\mid \int_{t}^{\infty}(s-t)^{N-1} h_{2}\left(t, x_{1}(s), x_{1}^{\prime}(s), . ., x_{1}^{\left(N_{1}\right)}(s), x_{2}(s), x_{2}^{\prime}(s), . ., x_{2}^{\left(N_{2}\right)}(s)\right)\right) d s \mid \\
& \leq K_{2}
\end{aligned}
$$

and

$$
\begin{aligned}
& \left.\mid \int_{t}^{\infty}(s-t)^{N-1-j} h_{1}\left(t, x_{1}(s), x_{1}^{\prime}(s), . ., x_{1}^{\left(N_{1}\right)}(s), x_{2}(s), x_{2}^{\prime}(s), . ., x_{2}^{\left(N_{2}\right)}(s)\right)\right) d s \mid \\
& \leq K_{1} \text {, } \\
& \left.\mid \int_{t}^{\infty}(s-t)^{N-1-j} h_{2}\left(t, x_{1}(s), x_{1}^{\prime}(s), . ., x_{1}^{\left(N_{1}\right)}(s), x_{2}(s), x_{2}^{\prime}(s), \ldots, x_{2}^{\left(N_{2}\right)}(s)\right)\right) d s \mid \\
& \leq K_{2}
\end{aligned}
$$

for every $t \geq T$ and $j=0,1,2, . ., N-1$.

As this is true for any pair $x_{1}, x_{2}$, we now define mapping $S=\left(S_{1}\left(x_{1}, x_{2}\right), S_{2}\left(x_{1}, x_{2}\right)\right)$ on $X \times X$ as

$$
\begin{gathered}
\left(S_{1}\left(x_{1}, x_{2}\right)\right)(t)=K_{1-} \\
\left.(-1)^{n_{1}} \int_{t}^{\infty} \frac{(s-t)^{n_{1}-1}}{\left(n_{1}-1\right) !} h_{1}\left(t, x_{1}(s), x_{1}^{\prime}(s), . ., x_{1}^{\left(N_{1}\right)}(s), x_{2}(s), x_{2}(s), . ., x_{2}^{\left(N_{2}\right)}(s)\right)\right) d s
\end{gathered}
$$

and

$$
\left(S_{2}\left(x_{1}, x_{2}\right)\right)(t)=K_{2}-
$$




$$
\left.(-1)^{n_{2}} \int_{t}^{\infty} \frac{(s-t)^{n_{2}-1}}{\left(n_{1}-1\right) !} h_{2}\left(t, x_{1}(s), x_{1}^{\prime}(s), . ., x_{1}^{\left(N_{1}\right)}(s), x_{2}(s), x_{2}^{\prime}(s), . ., x_{2}^{\left(N_{2}\right)}(s)\right)\right) d s
$$

for every $t \geq T$.

It is not difficult to notice here that the map $S$, is a self map from $Y=X \times X$ to itself and is well defined because $S_{1}(X \times X) \subseteq X$ and $S_{2}(X \times X) \subseteq X$. We apply the Shauder's theorem and show that $S$ has a fixed point pair. We first show that $S_{1} Y$ is relatively compact. We do this for $S_{1}$ and an analogous proof follows for $S_{2}$ also to show that $S_{2} Y$ is relatively compact, which we exclude. After applying the compactness criterion Lemma 2.6 given earlier, it suffices to show that each one of the sets $\left(S_{1} Y\right)^{(k)}, k=0,1, . ., n_{1}-1$, are uniformly bounded, equicontinuous and equiconvergent at $\infty$.

Since $S_{1} Y \subset Y$, we obviously have $\left(S_{1}\left(x_{1}, x_{2}\right)\right)^{(k)}<K_{1}$ for $k=0,1, . ., n_{1}-1$ for all $\left(x_{1}, x_{2}\right) \in Y$. So $\left(S_{1} X\right)^{(k)}$ for $k=0,1, \ldots, n_{1}-1$ are uniformly bounded.

Moreover for some $t \geq T^{\prime} \geq T$, we have

$$
\begin{aligned}
& \left|S_{1}\left(x_{1}(t), x_{2}(t)\right)-K_{1}\right|= \\
& \left.\mid(-1)^{n_{1}} \int_{t}^{\infty} \frac{(s-t)^{n_{1}-1}}{\left(n_{1}-1\right) !} h_{1}\left(t, x_{1}(s), x_{1}^{\prime}(s), . ., x_{1}^{\left(N_{1}\right)}(s), x_{2}(s), x_{2}^{\prime}(s), \ldots, x_{2}^{\left(N_{2}\right)}(s)\right)\right) d s \mid \\
& \left.\leq \int_{t}^{\infty}(s-T) \frac{(s-t)^{n_{1}-1}}{\left(n_{1}-1\right) !} \mid h_{1}\left(t, x_{1}(s), x_{1}^{\prime}(s), . ., x_{1}^{\left(N_{1}\right)}(s), x_{2}(s), x_{2}^{\prime}(s), . ., x_{2}^{\left(N_{2}\right)}(s)\right)\right) \mid d s \\
& \leq \sum_{l=1}^{N_{1}+N_{2}} \Theta_{1 l} \int_{t}^{\infty} \frac{(s-t)^{n_{1}-1}}{\left(n_{1}-1\right) !} p_{1 l}(s) d s+\int_{t}^{\infty} \frac{(s-t)^{n_{1}-1}}{\left(n_{1}-1\right) !} r_{1}(s) d s
\end{aligned}
$$

for all $\left(x_{1}, x_{2}\right) \in Y$.

and

$$
\begin{gathered}
\left|\left(S_{1}\right)^{(k)}\left(x_{1}(t), x_{2}(t)\right)\right|= \\
\left.\mid(-1)^{n_{1}} \int_{t}^{\infty} \frac{(s-t)^{n_{1}-1-k}}{\left(n_{1}-1-k\right) !} h_{1}\left(t, x_{1}(s), x_{1}^{\prime}(s), . ., x_{1}^{\left(N_{1}\right)}(s), x_{2}(s), x_{2}^{\prime}(s), . ., x_{2}^{\left(N_{2}\right)}(s)\right)\right) d s \mid \\
\left.\leq \int_{t}^{\infty}(s-T) \frac{(s-t)^{n_{1}-1-k}}{\left(n_{1}-1-k\right) !} \mid h_{1}\left(t, x_{1}(s), x_{1}(s), . ., x_{1}^{\left(N_{1}\right)}(s), x_{2}(s), x_{2}(s), \ldots, x_{2}^{\left(N_{2}\right)}(s)\right)\right) \mid d s \\
\leq \sum_{l=1}^{N_{1}+N_{2}} \Theta_{1 l} \int_{t}^{\infty} \frac{(s-t)^{n_{1}-1-k}}{\left(n_{1}-1-k\right) !} p_{1 l}(s) d s+\int_{t}^{\infty} \frac{(s-t)^{n_{1}-1-k}}{\left(n_{1}-1-k\right) !} r_{1}(s) d s
\end{gathered}
$$

for $k=1,2, \ldots, n_{1}-1$. So, by using (3.2) and suitably choosing a large $T^{\prime}$ such that $\int_{t}^{\infty} \frac{(s-t)^{n_{1}-1-k}}{\left(n_{1}-1-k\right) !} p_{1 l}(s) d s<$ $\frac{\epsilon}{2\left(N_{1}+N_{2}\right) \max \left\{\Theta_{1 l}\right\}}, l=1,2, . ., N_{1}+N_{2}$ and $\int_{t}^{\infty} \frac{(s-t)^{n_{1}-1-k}}{\left(n_{1}-1-k\right) !} r_{1}(s) d s<\frac{\epsilon}{2}$ for all $t>T^{\prime}$ and for $k=1,2, . ., n_{1}-1$, we see that the right hand side of the inequality can be made close to zero, therefore we conclude that $S_{1} Y$ is equiconvergent at $\infty$.

Now by using (3.5) for any $\left(x_{1}, x_{2}\right) \in Y$, for every $t_{1}, t_{2}$ with $T^{\prime} \leq t_{1}<t_{2}$ and for $k<n_{1}-1$, we see that

$$
\left|\left(S_{1}\right)^{(k)}\left(x_{1}\left(t_{2}\right), x_{2}\left(t_{2}\right)\right)-\left(S_{1}\right)^{(k)}\left(x_{1}\left(t_{1}\right), x_{2}\left(t_{1}\right)\right)\right|=
$$




$$
\begin{gathered}
\left.\mid K_{1}-(-1)^{n_{1}} \int_{t_{2}}^{\infty} \frac{\left(s-t_{2}\right)^{n_{1}-1-k}}{\left(n_{1}-1-k\right) !} h_{1}\left(t, x_{1}(s), x_{1}^{\prime}(s), \ldots, x_{1}^{\left(N_{1}\right)}(s), x_{2}(s), x_{2}^{\prime}(s), \ldots, x_{2}^{\left(N_{2}\right)}(s)\right)\right) d s \\
\left.-K_{1}+(-1)^{n_{1}} \int_{t_{1}}^{\infty} \frac{\left(s-t_{1}\right)^{n_{1}-1-k}}{\left(n_{1}-1-k\right) !} h_{1}\left(t, x_{1}(s), x_{1}^{\prime}(s), \ldots, x_{1}^{\left(N_{1}\right)}(s), x_{2}(s), x_{2}^{\prime}(s), \ldots, x_{2}^{\left(N_{2}\right)}(s)\right)\right) d s \mid \\
\left.=\mid \int_{t_{2}}^{\infty}\left[\int_{r}^{\infty} \frac{\left(s-t_{1}\right)^{n_{1}-2-k}}{\left(n_{1}-2-k\right) !} h_{1}\left(t, x_{1}(s), x_{1}(s), \ldots, x_{1}^{\left(N_{1}\right)}(s), x_{2}(s), x_{2}(s), . ., x_{2}^{\left(N_{2}\right)}(s)\right)\right) d s\right] d r \\
\left.-\int_{t_{1}}^{\infty}\left[\int_{r}^{\infty} \frac{\left(s-t_{2}\right)^{n_{1}-2-k}}{\left(n_{1}-2-k\right) !} h_{1}\left(t, x_{1}(s), x_{1}^{\prime}(s), \ldots, x_{1}^{\left(N_{1}\right)}(s), x_{2}(s), x_{2}(s), . ., x_{2}^{\left(N_{2}\right)}(s)\right)\right) d s\right] d r \mid \\
\left.=\mid \int_{t_{1}}^{t_{2}}\left[\int_{r}^{\infty} \frac{(s-r)^{n_{1}-2-k}}{\left(n_{1}-2-k\right) !} h_{1}\left(t, x_{1}(s), x_{1}^{\prime}(s), \ldots, x_{1}^{\left(N_{1}\right)}(s), x_{2}(s), x_{2}^{\prime}(s), \ldots, x_{2}^{\left(N_{2}\right)}(s)\right)\right) d s\right] d r \mid \\
\left.=\int_{t_{1}}^{t_{2}}\left[\int_{r}^{\infty} \frac{(s-r)^{n_{1}-2-k}}{\left(n_{1}-2-k\right) !} \mid h_{1}\left(t, x_{1}(s), x_{1}^{\prime}(s), \ldots, x_{1}^{\left(N_{1}\right)}(s), x_{2}(s), x_{2}^{\prime}(s), \ldots, x_{2}^{\left(N_{2}\right)}(s)\right)\right) \mid d s\right] d r \\
\leq \sum_{l=1}^{N_{1}+N_{2}} \Theta_{1 l}^{t_{2}}\left[\int_{t_{1}}^{\infty}\left[\frac{(s-r)^{n_{1}-2-k}}{\left(n_{1}-2-k\right) !} p_{1 l}(s) d s\right] d r+\int_{t_{1}}^{t_{2}}\left[\int_{r}^{\infty} \frac{(s-r)^{n_{1}-2-k}}{\left(n_{1}-2-k\right) !} r_{1}(s) d s\right] d r .\right.
\end{gathered}
$$

Also for $k=n_{1}-1$ we have

$$
\begin{gathered}
\left|\left(s_{1}\right)^{\left(n_{1}-1\right)}\left(x_{1}\left(t_{2}\right), x_{2}\left(t_{2}\right)\right)-\left(s_{1}\right)^{\left(n_{1}-1\right)}\left(x_{1}\left(t_{1}\right), x_{2}\left(t_{1}\right)\right)\right|= \\
\left.\mid K_{1}-(-1)^{n_{1}} \int_{t_{2}}^{\infty} h_{1}\left(t, x_{1}(s), x_{1}^{\prime}(s), \ldots, x_{1}^{\left(N_{1}\right)}(s), x_{2}(s), x_{2}^{\prime}(s), \ldots, x_{2}^{\left(N_{2}\right)}(s)\right)\right) d s \\
\left.-K_{1}+(-1)^{n_{1}} \int_{t_{1}}^{\infty} h_{1}\left(t, x_{1}(s), x_{1}(s), \ldots, x_{1}^{\left(N_{1}\right)}(s), x_{2}(s), x_{2}(s), \ldots, x_{2}^{\left(N_{2}\right)}(s)\right)\right) d s \mid \\
\left.=\mid \int_{t_{2}}^{\infty}\left[\int_{r}^{\infty} h_{1}\left(t, x_{1}(s), x_{1}(s), \ldots, x_{1}^{\left(N_{1}\right)}(s), x_{2}(s), x_{2}(s), \ldots, x_{2}^{\left(N_{2}\right)}(s)\right)\right) d s\right] d r \\
\left.-\int_{t_{1}}^{\infty}\left[\int_{r}^{\infty} h_{1}\left(t, x_{1}(s), x_{1}^{\prime}(s), \ldots, x_{1}^{\left(N_{1}\right)}(s), x_{2}(s), x_{2}^{\prime}(s), \ldots, x_{2}^{\left(N_{2}\right)}(s)\right)\right) d s\right] d r \mid \\
\left.=\mid \int_{t_{1}}^{t_{2}}\left[\int_{r}^{\infty} h_{1}\left(t, x_{1}(s), x_{1}^{\prime}(s), \ldots, x_{1}^{\left(N_{1}\right)}(s), x_{2}(s), x_{2}^{\prime}(s), \ldots, x_{2}^{\left(N_{2}\right)}(s)\right)\right) d s\right] d r \mid \\
\left.=\int_{t_{1}}^{t_{2}}\left[\int_{r}^{\infty} \mid h_{1}\left(t, x_{1}(s), x_{1}^{\prime}(s), \ldots, x_{1}^{\left(N_{1}\right)}(s), x_{2}(s), x_{2}^{\prime}(s), \ldots, x_{2}^{\left(N_{2}\right)}(s)\right)\right) \mid d s\right] d r \\
\leq \sum_{l=1}^{N_{1}+N_{2}} \Theta_{1 l} \int_{t_{1}}^{t_{2}}\left[\int_{r}^{\infty} p_{1 l}(s) d s\right] d r+\int_{t_{1}}^{t_{2}}\left[\int_{r}^{\infty} r_{1}(s) d s\right] d r .
\end{gathered}
$$


By referring to condition (3.2) we let $A=\int_{r}^{\infty} \frac{(s-r)^{n_{1}-2}}{\left(n_{1}-2\right) !} p_{1 l}(s) d s$ and $\int_{r}^{\infty} \frac{(s-r)^{n_{1}-2}}{\left(n_{1}-2\right) !} r_{1}(s) d s$. Now, by choosing $\left|t_{2}-t_{1}\right|<\frac{\epsilon}{2 A\left(N_{1}+N_{2}\right) \max \left\{\Theta_{1 l}\right\}}$ we conclude that $S_{1} Y$ is equicontinuous. Therefore, $S_{1} Y$ is relatively compact. A similar proof can be given to show that $S_{2} Y$ is relatively compact. Therefore, $S Y$ is relatively compact. To apply Schauder's theorem, the mapping $S$ has to be continuous. Consider $\left(x_{1 v}, x_{2 v}\right)$ to be a random sequence in $Y$, converging to $\left(x_{1}, x_{2}\right)$ under the norm defined before. From (3.5) we have

$$
\left.\mid h_{i}\left(t, x_{1 v}(t), x_{1 v}^{\prime}(t), \ldots, x_{1 v}^{\left(N_{1}\right)}(t), x_{2 v}(t), x_{2 v}^{\prime}(t), . ., x_{2 v}^{\left(N_{2}\right)}(t)\right)\right) \mid \leq \Theta_{i} p_{i}(t)+r_{i}(t)
$$

for every $t \geq T$ and for all $v \in N$.

Now, due to the Lebesgue's dominated convergence theorem we have

$$
\begin{aligned}
\left.\lim _{v \rightarrow \infty} \int_{t}^{\infty} \frac{(s-t)^{n_{i}-1}}{\left(n_{i}-1\right) !} h_{i}\left(s, x_{1 v}(s), x_{1 v}^{\prime}(s), . ., x_{1 v}^{\left(N_{1}\right)}(s), x_{2 v}(s), x_{2 v}^{\prime}(s), . ., x_{2 v}^{\left(N_{2}\right)}(s)\right)\right) d s \\
\left.\quad=\int_{t}^{\infty} \frac{(s-t)^{n_{i}-1}}{\left(n_{i}-1\right) !} h_{i}\left(s, x_{1}(s), x_{1}^{\prime}(s), . ., x_{1}^{\left(N_{1}\right)}(s), x_{2}(s), x_{2}^{\prime}(s), . ., x_{2}^{\left(N_{2}\right)}(s)\right)\right) d s .
\end{aligned}
$$

This proves the pointwise convergence i.e

$$
\lim _{v \rightarrow \infty}\left(S\left(x_{1 v}, x_{2 v}\right)\right)(t)=\left(S\left(x_{1}, x_{2}\right)\right)(t) .
$$

Now, consider any random subsequence $\left(u_{\mu_{1}}, u_{\mu_{2}}\right)$ of $S\left(x_{1 v}, x_{2 v}\right)$. The relatively compactness of $S Y$ guarantees the existence of a subsequence $\left(\eta_{\lambda_{1}}, \eta_{\lambda_{2}}\right)$ of $\left(u_{\mu_{1}}, u_{\mu_{2}}\right)$ and a pair of functions $\left(\eta_{1}, \eta_{2}\right)$ in $E \operatorname{such}$ that $\left(v_{\lambda_{1}}, v_{\lambda_{2}}\right)$ converges uniformly to $\left(v_{1}, v_{2}\right)$. So

$$
\lim _{v \rightarrow \infty}\left(S\left(x_{1 v}, x_{2 v}\right)\right)(t)=\left(S\left(x_{1}, x_{2}\right)\right)(t)=\left(\eta_{1}, \eta_{2}\right)
$$

for all $t \geq T$ under the sup-norm. Therefore $S$ is continuous.

Thus $S$ satisfies all the assumptions of Schauder's theorem, therefore $S$ has a fixed point pair $\left(x_{1}, x_{2}\right) \in Y$ such that $S_{1}\left(x_{1}, x_{2}\right)=x_{1}$ and $S_{2}\left(x_{1}, x_{2}\right)=x_{2}$. That implies

$$
\left.x_{i}(t)=K_{i}-(-1)^{n_{i}} \int_{t}^{\infty} \frac{(s-t)^{n_{i}-1}}{\left(n_{i}-1\right) !} h_{i}\left(s, x_{1}(s), x_{1}^{\prime}(s), . ., x_{1}^{\left(N_{1}\right)}(s), x_{2}(s), x_{2}^{\prime}(s), \ldots, x_{2}^{\left(N_{2}\right)}(s)\right)\right) d s .
$$

So by differentiating the above equation we see that

$$
\left.x_{i}^{\left(n_{i}\right)}(t)+h_{i}\left(t, x_{1}(t), x_{1}^{\prime}(t), . ., x_{1}^{\left(N_{1}\right)}(t), x_{2}(t), x_{2}^{\prime}(t), . ., x_{2}^{\left(N_{2}\right)}(t)\right)\right)=0
$$

for all $t \geq T$ and also $x_{i} \rightarrow K_{i}$ as $t \rightarrow \infty$.

For arbitrary large values of $t$ we see that the solutions $x_{i} \rightarrow K_{i}, i=1,2$. This also means that the solutions can not have arbitrarily large zeros, so the solution is non-oscillatory.

Theorem 3.2. Let $K_{1}>0, K_{2}>0$ be given and fixed. Assume that $h_{1}$ and $h_{2}$ are functions from $\mathbb{R}^{+} \times \mathbb{R}^{N_{1}+N_{2}}$ to $\mathbb{R}^{+}$and satisfy

$$
\int_{t_{0}}^{\infty} t^{n-1} h_{i}\left(t, z_{1}, z_{2}, \ldots z_{N_{1}+N_{2}}\right) d t \leq K_{i}
$$

for $i=1,2, n=\max \left\{n_{1}, n_{2}\right\}$, any $z_{i} \in \mathbb{R}$ and let

$$
\begin{aligned}
& \left|h_{1}\left(t, z_{1}, . ., z_{N_{1}+N_{2}}\right)-h_{1}\left(t, \widehat{z_{1}}, \ldots, \widehat{z_{N_{1}+N_{2}}}\right)\right| \leq a(t)\left(\sum_{i=1}^{N_{1}+N_{2}}\left|z_{i}-\widehat{z_{i}}\right|\right), \\
& \left|h_{2}\left(t, z_{1}, \ldots, z_{N_{1}+N_{2}}\right)-h_{1}\left(t, \widehat{z_{1}}, \ldots, \widehat{z_{N_{1}+N_{2}}}\right)\right| \leq b(t)\left(\sum_{i=1}^{N_{1}+N_{2}}\left|z_{i}-\widehat{z_{i}}\right|\right),
\end{aligned}
$$


where $a(t), b(t)$ be continuous functions from $\mathbb{R}^{+}$to $\mathbb{R}^{+}$such that

$$
\begin{aligned}
& \int_{t_{0}}^{\infty} t^{n-1} a(t) d t<1 / 2, \\
& \int_{t_{0}}^{\infty} t^{n-1} b(t) d t<1 / 2 .
\end{aligned}
$$

Then the system (1.5) has a unique solution pair $\left\{x_{1}, x_{2}\right\}$ on the interval $\left(t_{0}, \infty\right)$ such that $x_{1} \rightarrow K_{1}$ and $x_{2} \rightarrow K_{2}$ asymptotically and therefore non-oscillatory.

Proof. Let $X=\left\{\left(x_{1}, x_{2}\right) \in(B)^{(N)}\left(\left[t_{0}, \infty\right)\right) \times(B)^{(N)}\left(\left[t_{0}, \infty\right)\right): 0 \leq\|x(t)\|_{N \times N} \leq K\right\}$

where $K=\max \left\{2 K_{1}, 2 K_{2}\right\}$.

We clearly see that the set $X$ is a closed subset of $(B)^{(N)}\left(\left[t_{0}, \infty\right)\right) \times(B)^{(N)}\left(\left[t_{0}, \infty\right]\right)$. It follows that $\left(X,\|\cdot\|_{N \times N}\right)$, where $\|\cdot\|_{N \times N}$ is the usual norm defined in (2.2), is a Banach Space.

Let us now define the operator $S=\left(S_{1}, S_{2}\right)$ on the set $X_{1}$ as follows

$$
\begin{aligned}
\left(S_{1}\left(x_{1}, x_{2}\right)\right)(t)= & K_{1}-(-1)^{n_{1}} \int_{t}^{\infty} \frac{(s-t)^{n_{1}-1}}{\left(n_{1}-1\right) !} \\
& \left.\times h_{1}\left(t, x_{1}(s), x_{1}^{\prime}(s), \ldots, x_{1}^{\left(N_{1}\right)}(s), x_{2}(s), x_{2}(s), \ldots, x_{2}^{\left(N_{2}\right)}(s)\right)\right) d s, \\
\left(S_{2}\left(x_{1}, x_{2}\right)\right)(t)= & K_{2}-(-1)^{n_{1}} \int_{t}^{\infty} \frac{(s-t)^{n_{2}-1}}{\left(n_{1}-1\right) !} \\
& \left.\times h_{2}\left(t, x_{1}(s), x_{1}^{\prime}(s), . ., x_{1}^{\left(N_{1}\right)}(s), x_{2}(s), x_{2}^{\prime}(s), \ldots, x_{2}^{\left(N_{2}\right)}(s)\right)\right) d s
\end{aligned}
$$

for every $t \geq t_{0}$.

The integral part in the right hand side of $S_{1}$ and $S_{2}$ is definitely convergent due to (3.8). Indeed for any given $\left(x_{1}, x_{2}\right) \in X$ and for every $t \geq t_{0}$,

$$
\left\|\left(S\left(x_{1}, x_{2}\right)\right)(t)\right\|_{N \times N}=\max \left\{\left\|\left(S_{1}\left(x_{1}, x_{2}\right)\right)(t)\right\|_{N},\left\|\left(S_{2}\left(x_{1}, x_{2}\right)\right)(t)\right\|_{N}\right\} \leq \max \left\{2 K_{1}, 2 K_{2}\right\} .
$$

Thus, we see that $S X \subseteq X$.

We show that this operator has a unique fixed point using the Banach's fixed point theorem. Clearly such a fixed point is a solution that we are looking for. So, it suffices to show that $S$ is a contraction.

Consider now,

$$
\begin{aligned}
& \left\|S_{1}\left(x_{1}, x_{2}\right)-S_{1}\left(\widehat{x}_{1}, \widehat{x}_{2}\right)\right\|= \\
& \left.\|(-1)^{n_{1}} \int_{t}^{\infty} \frac{(s-t)^{n_{1}-1}}{\left(n_{1}-1\right) !} h_{1}\left(t, x_{1}(s), x_{1}^{\prime}(s), \ldots, x_{1}^{\left(N_{1}\right)}(s), x_{2}(s), x_{2}^{\prime}(s), \ldots, x_{2}^{\left(N_{2}\right)}(s)\right)\right) d s \\
& \left.-(-1)^{n_{1}} \int_{t}^{\infty} \frac{(s-t)^{n_{1}-1}}{\left(n_{1}-1\right) !} h_{1}\left(t, \widehat{x}_{1}(s), \widehat{x}_{1}^{\prime}(s), \ldots, \widehat{x}_{1}^{\left(N_{1}\right)}(s), \widehat{x}_{2}(s), \widehat{x}_{2}^{\prime}(s), \ldots, \widehat{x}_{2}^{\left(N_{2}\right)}(s)\right)\right) d s \| \\
& \left.\leq \int_{t}^{\infty} \frac{(s-t)^{n_{1}-1}}{\left(n_{1}-1\right) !} \| h_{1}\left(t, x_{1}(s), x_{1}^{\prime}(s), . ., x_{1}^{\left(N_{1}\right)}(s), x_{2}(s), x_{2}^{\prime}(s), . ., x_{2}^{\left(N_{2}\right)}(s)\right)\right)- \\
& \left.h_{1}\left(t, \widehat{x}_{1}(s), \widehat{x}_{1}^{\prime}(s), \ldots, \widehat{x}_{1}^{\left(N_{1}\right)}(s), \widehat{x}_{2}(s), \widehat{x}_{2}^{\prime}(s), \ldots, \widehat{x}_{2}^{\left(N_{2}\right)}(s)\right)\right) \| d s \\
& \leq\left[\int_{t}^{\infty} a(s) d s\right]\left\|\left(x_{1}, x_{2}\right)\right\|_{N \times N} .
\end{aligned}
$$


In similar fashion we can show

$$
\left\|S_{2}\left(x_{1}, x_{2}\right)-S_{2}\left(\widehat{x}_{1}, \widehat{x}_{2}\right)\right\| \leq\left[\int_{t}^{\infty} b(s) d s\right]\left\|\left(x_{1}, x_{2}\right)\right\|_{N \times N} .
$$

Now taking (3.10) in to consideration, we see that $S$ is a contraction, hence by contraction principle $S$ has a unique fixed point pair. This fixed point pair $x_{1}, x_{2}$ satisfies the equation

$$
\left.x_{i}(t)=K_{i}-(-1)^{n_{i}} \int_{t}^{\infty} \frac{(s-t)^{n_{i}-1}}{\left(n_{i}-1\right) !} h_{i}\left(t, x_{1}(s), x_{1}^{\prime}(s), . ., x_{1}^{\left(N_{1}\right)}(s), x_{2}(s), x_{2}^{\prime}(s), \ldots, x_{2}^{\left(N_{2}\right)}(s)\right)\right) d s
$$

for $i=1$, 2. Differentiating the above equation $n_{i}$ times gives us

$$
x_{i}^{\left(n_{i}\right)}(t)+h_{i}\left(t, x_{1}, x_{1}^{\prime}, \ldots, x_{1}^{\left(N_{1}\right)}, x_{2}, x_{2}^{\prime}, . ., x_{2}^{\left(N_{2}\right)}\right)=0
$$

for $i=1,2$.

Therefore these fixed points $x_{1}$ and $x_{2}$ definitely satisfy the differential system (1.5) and $x_{i} \rightarrow K_{i}$ as $t \rightarrow \infty$ for $i=1,2$. This is a direct consequence by the definition of the integral operator because differentiating the integral operator gives back the system of differential equations and the presence of constants ensures the asymptotic property for large values of $t$.

Example 3.3. consider the system of differential equations

$$
\begin{aligned}
& y_{1}^{\prime \prime}(t)+p_{1}(t)\left|y_{2}(t)\right|^{\gamma_{1}}+p_{2}(t)\left|y_{1}^{\prime}(t)\right|^{\delta_{1}}=0, \\
& y_{2}^{\prime \prime}(t)+q_{1}(t)\left|y_{1}(t)\right|^{\gamma_{2}}+q_{2}(t)\left|y_{2}^{\prime}(t)\right|^{\delta_{2}}=0,
\end{aligned}
$$

where $p(t), q(t)$ are non-negative continuous real valued functions such that

$$
\begin{aligned}
& \int_{t_{0}}^{\infty} t p_{i}(t)<\infty, \\
& \int_{t_{0}}^{\infty} t q_{i}(t)<\infty
\end{aligned}
$$

and $\gamma_{i}, \delta_{i}$ are positive constants. Let $T$ be a point with $T \geq t_{0}$ and suppose there exist positive constants $K_{1}$ and $K_{2}$ such that

$$
\begin{aligned}
& {\left[\int_{T}^{\infty}(s-T) p_{1}(s) d s\right]\left(\max \left\{2 K_{1}, 2 K_{2}\right\}\right)^{\gamma_{1}}+\left[\int_{T}^{\infty}(s-T) p_{2}(s) d s\right]\left(\max \left\{2 K_{1}, 2 K_{2}\right\}\right)^{\delta_{1}}} \\
& \leq K_{1}, \\
& {\left[\int_{T}^{\infty}(s-T) q_{1}(s) d s\right]\left(\max \left\{2 K_{1}, 2 K_{2}\right\}\right)^{\gamma_{2}}+\left[\int_{T}^{\infty}(s-T) q_{2}(s) d s\right]\left(\max \left\{2 K_{1}, 2 K_{2}\right\}\right)^{\delta_{2}}} \\
& \leq K_{2}
\end{aligned}
$$

and

$$
\begin{aligned}
& {\left[\int_{T}^{\infty} p_{1}(s) d s\right]\left(\max \left\{2 K_{1}, 2 K_{2}\right\}\right)^{\gamma_{1}}+\left[\int_{T}^{\infty} p_{2}(s) d s\right]\left(\max \left\{2 K_{1}, 2 K_{2}\right\}\right)^{\delta_{1}} \leq K_{1},} \\
& {\left[\int_{T}^{\infty} q_{1}(s) d s\right]\left(\max \left\{2 K_{1}, 2 K_{2}\right\}\right)^{\gamma_{2}}+\left[\int_{T}^{\infty} q_{2}(s) d s\right]\left(\max \left\{2 K_{1}, 2 K_{2}\right\}\right)^{\delta_{2}} \leq K_{2} .}
\end{aligned}
$$


We see that the system (3.11) satisfies conditions (3.2), (3.3), (3.4) of Theorem 3.1. By applying Theorem 3.1, existence of a non-oscillating solution to (3.11) such that $y_{i} \rightarrow K_{i}$ for $i=1,2$ as $t \rightarrow \infty$ is guaranteed. For the special case where $\gamma_{i}=\delta_{i}=2$, for $i=1,2$ and $K_{1}=K_{2}=1 / 2$, these conditions boil down to finding if functions $p_{i}$ and $q_{i}$ are such that

$$
\begin{aligned}
& {\left[\int_{T}^{\infty}(s-T) p_{1}(s) d s\right]+\left[\int_{T}^{\infty}(s-T) p_{2}(s) d s\right] \leq 1 / 2,} \\
& {\left[\int_{T}^{\infty}(s-T) q_{1}(s) d s\right]+\left[\int_{T}^{\infty}(s-T) q_{2}(s) d s\right] \leq 1 / 2}
\end{aligned}
$$

and

$$
\begin{aligned}
& {\left[\int_{T}^{\infty} p_{1}(s) d s\right]+\left[\int_{T}^{\infty} p_{2}(s) d s\right] \leq 1 / 2,} \\
& {\left[\int_{T}^{\infty} q_{1}(s) d s\right]+\left[\int_{T}^{\infty} q_{2}(s) d s\right] \leq 1 / 2}
\end{aligned}
$$

for some $T>0$. We note here that fixing $T=2, p_{1}(t)=e^{-t} / 2, p_{2}(t)=e^{-t-2} / 2$ and $q_{1}(t)=e^{-t-2} / 2, q_{2}(t)=e^{-t} / 2$ satisfy the above inequalities.

Acknowledgment and Dedication: We dedicate this work to the Founder Chancellor of Sri Sathya Sai Institute of Higher Learning, Bhagawan Sri Sathya Sai Baba.

This work is partially funded by National Board of Higher Mathematics, INDIA (Grant No: 2/48(12)/2009-R\&D II/2808).

Conflict of interest: Authors have no conflict of interest.

\section{References}

[1] R Ma, Existence of positive radial solutions for elliptic systems, J.Math.Anal.App. 201(1996), 375-386.

[2] B V K Bharadwaj and Pallav Kumar Baruah, Asymptotically Polynomial type solutions for some 2-dimensional coupled nonlinear ODEs, The Journal of Mathematics and Computer Science, Vol 14(2015), no 3, 211-221.

[3] B V K Bharadwaj and Pallav Kumar Baruah, Existence of a Non-Oscillating solution for a second order nonlinear ODE, Communications in Mathematics and Applications, Vol.6, No 2, 41-47.

[4] B.V.K. Bharadwaj and Pallav Kumar Baruah, Existence of a Non-Oscillating solution for a System of Nonlinear ODEs, International Journal of Advances in Mathematics, Volume 2018, Number 4, Pages 34-43, 2018.

[5] B.V.K. Bharadwaj and Pallav Kumar Baruah, Asymptotically polynomial type solutions for 2-dimensional coupled non-linear ODEs with derivative terms, accepted for publication in International Journal of Dynamical Systems and Differential Equationsm Inderscience Publishers.

[6] Ch.G. Philos, I.K. Purnaras and P.Ch. Tsamatos, Asymptotic to polynomial solutions for nonlinear differential equations, Nonlinear Analysis. 59(2004), 1157-1179

[7] S. Dube and B. Mingarelli, Note on a non-oscillation theorem of Atkinson, EJDE, Vol 2004(2004), No. 22, 1-6.

[8] G. J. Butler, On the non-oscillatory behavior of a second order nonlinear differential equation, Annali Mat. Pura Appl. (4) 105 (1975), 73-92.

[9] E. Wahlen, Positive Solutions of second order differential equations, Nonlinear Analysis 58 (2004), 359-366.

[10] Chritos G Philos, Panagiotis Ch Tsamatos, Solutions approaching polynomials at infinity to nonlinear ordinary differential equations, EJDE, 2005(2005), no. 79, 1-25.

[11] Orlando Lopes, Uniqueness of a Symmetric Positive Solution to an ODE System, EJDE, 2009(2009), no 162, 1-8.

[12] B. Banarjee, Magneto-hydrodynamic flow between two horizontel plates in a rotating system, lower plate being a stretched sheet, Trans. ASME 50 (1983), 470-480.

[13] John R Graef and Toufik Moussaoui, Positive solutions of a system of coupled second order equations with three point boundary conditions, Rocky mountain journal of mathematics, Vol 42, No 4, 1169-1182. 
[14] J Henderson, S K Ntouyas, Positive Solutions for Systems of nth Order Three-point Nonlocal Boundary Value Problems, Electronic journal of qualitative theory of differential equations, 2007, No 18, 1-12. 